Iranian Journal of Pathology ISSN: 2345-3656

\title{
High Expression of Sphingosine Kinase 1 in Estrogen and Progesterone Receptors-Negative Breast Cancer
}

\author{
Azadeh-Sadat Nazouri ${ }^{1,2}$, Ommolbanin Asadpour ${ }^{3}$, Shahriar Dabiri ${ }^{2 *}$, Bahram Pourseyedi ${ }^{4}$, \\ Mohamad Reaza Lashkarizadeh $^{4}$, Hamid Zianalinejad ${ }^{4}$
}

1. Dept. of Biology, Science Faculty, Shahid Beheshti University, Tehran, Iran

2. Pathology and Stem Cell Research Center, Pathology Department, Afzalipour School of Medicine, Kerman University of Medical Sciences, Kerman, Iran

3. Dept. of Medical Biotechnology, Faculty of Medical Sciences, Tarbiat Modares University, Tehran, Iran 4. Surgery Department, Kerman University of Medical Sciences, Kerman, Iran

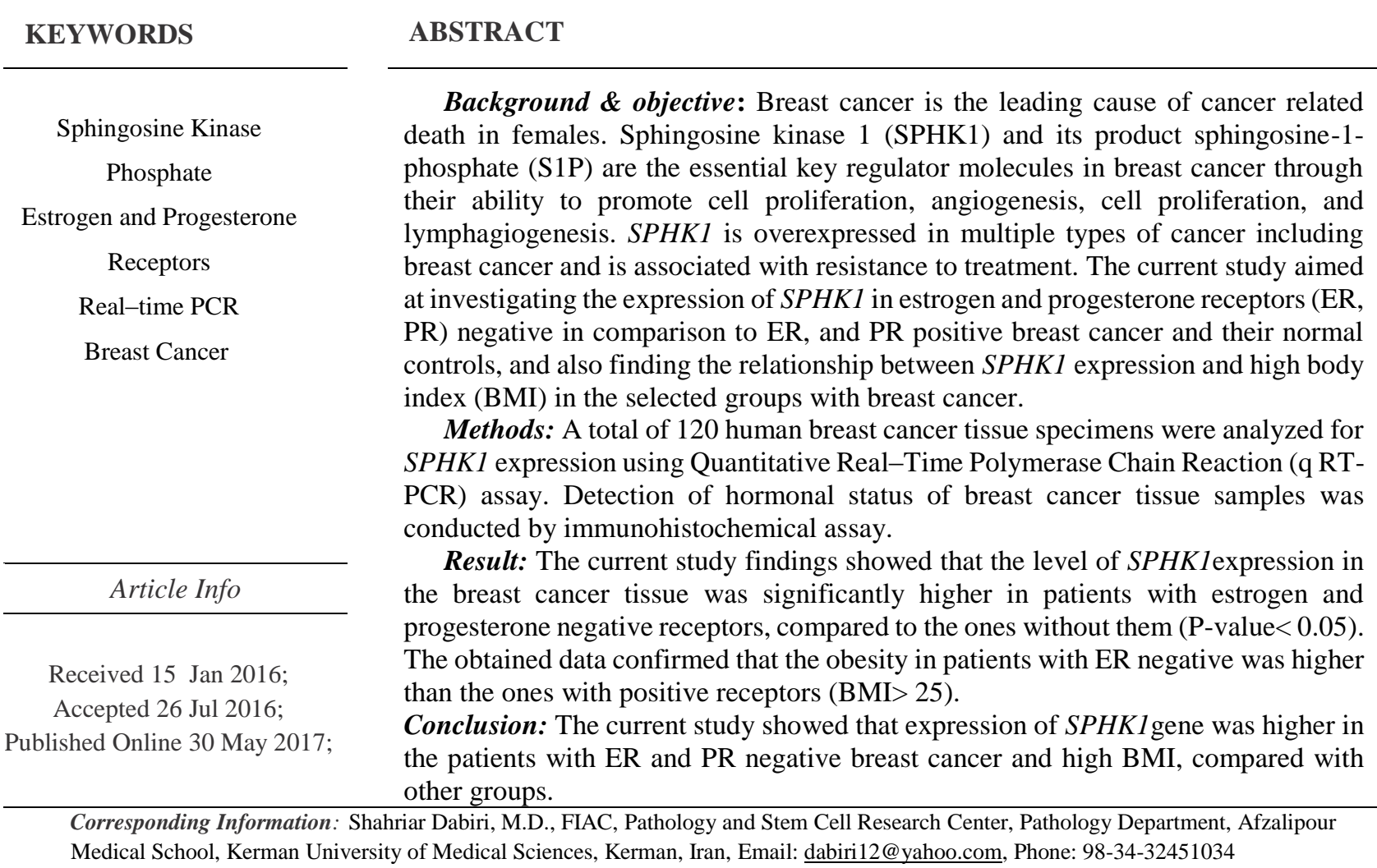

Copyright @ 2017, IRANIAN JOURNAL OF PATHOLOGY. This is an open-access article distributed under the terms of the Creative Commons Attribution-noncommercial 4.0 International License which permits copy and redistribute the material just in noncommercial usages, provided the original work is properly cited.

\section{Introduction}

Breast cancer is one of the most common cancers and is the leading cause of cancer-related death among females worldwide including Iran (1); $15 \%$ to $20 \%$ of the risk factors of developing this cancer is associated with genetics(2). The prevalence of breast cancer in Iran is among the females in the age range of 15 to 84 years; the age range of the participantswas 40 to 49 years. In the Iranian population, $77 \%$ and less than $5 \%$ of breast cancers are infiltrative ductal and lobular carcinoma, respectively (3). Different parameters such as tumor size, lymph node (LN) status, histological type and grade, status of hormonal receptors(ER and PR), and Her-2/ neu status are used for the prognosis of breast cancer.ER and PR expression is observed in more than $70 \%$ of breast cancer cases. Studies determined that the expression of ER and $\mathrm{PR}$ is associated with better response to hormone therapy $(4,5)$. As such patients lack estrogen and progesterone receptors, they are resistant to treatment that target these receptors and associated with poor prognosis (6). Therefore, finding new molecules, signaling pathways, and development of new therapies are important goals to treat breast cancer.Sphingosine-1-phosphate (S1P), ceramide, and sphingosine known as sphingolipid metabolites 
play different roles in molecular signaling of essential biological processes and the balance between them determines the cell fate; it means that when the balance goes towards ceramide and sphingosine, a cell is selected for death pathways and if the levels of S1P increase, a cell goes toward the survival and proliferation pathways. Hence, it is suggested that the perfect setting of these metabolites can affect the treatment of cancer ( 7 , 8).The balance is set by several regulators. Sphingosine kinases (SphKs) are introduced as unique regulators that produce S1P and decrease other metabolites (9).Anti-apoptotic lipid, S1P, is synthesized by SphKs, especially Sphingosine kinase 1 (SPHK1).Two distinct is of orms are known for SphKs; SPHK1 that regulates the essential processes of cancer progression, and SPHK2 that is much less known about its biological actions in cancer. High expression of SPHK1 is detected in multiple types of cancers, which can be associated with tumor angiogenesis and resistance to radiation and chemotherapy(10).SPHK1, as a cytosolic enzyme, is stimulated by binding multiple growth factors through their tyrosine kinase receptors (RTKs) to produce higher S1P $(10,11)$. Therefore, S1P function, both as an intracellular second messenger (12) and as specific ligand, activates $\mathrm{G}$ protein-coupled receptors (GPCRs) (9), and their downstream signaling to induce transactivation of various RTKs (10). SPHK1 has an important role in activation of cell proliferation, inflammatory response, migration and dysfunction in apoptosis, and its expression in tumor cells can lead to tumor growth, drug resistant, oncogenic transformation, neovascularization of tumors, and metastatic spread (13). SPHK1 was found from rat renal cells for the first time. It is located on chromosome $17 \mathrm{q} 25.2$ and has eight exons. Its expression was shown in multiple tissues such as brain, heart, lungs, spleen, and hematopoietic immune system (14). Evidence determined that SPHKl has oncogenic manner, which highlighted it as oncogenic enzyme (15), and can be a chemotherapeutic target because of its oncogenic characteristic and high expression of this gene and its product, S1P depends on many growth factors, which leads this gene and S1P toward stimulatory effects on tumor angiogenesis and cell motility that are critical for metastasis (10). It is overexpressed in many cancers such as lungs, colon, prostate (16), acute leukemia (17), clear cell renal carcinoma (18), and ovarian cancer (19). Overexpression of SPHK1 required to transform NIH3T3 fibroblasts to tumor phenotype in nude mice (15); and also the upregulation of this enzyme is associated with erythroleukemia (20). Inhibition of this gene reduces cell growth in triple negative breast cancer and clear cell renal carcinoma (ccRCC) (18), and decreases LN metastasis and tumor size (21). The current study aimed at investigating whether SPHKl is expressed highly in ER and PR negative compared to ER and PR positive breast cancers and their normal tissue, and also the relationship of this expression with BMI in patients with breast cancer.

\section{Materials and Methods}

The current case-control study selected 120 formalin fixed, paraffin embedded (FFPE) samples of patients aged 30 to 70 years from pathology wards of hospitals in Kerman, Iran, from 2010 to 2015.To calculate BMI, patients' demographic were recorded. Samples were collected after the approval of study protocol by the local ethical committee, and all cases signed the informed consent.

The 120 samples were divided into four groups of 30. Tumor groups included ER negative and ER positive breast cancer and the control groups including the normal tissue of selected tumor specimens. Immunohistochemistry (IHC) staining for ER and PR was performed by Dako Kit (Denmark).

\section{RNA extraction from FFPE tissue}

Three sections of 10-uM thick of each paraffin block were cut with microtome to study mRNA gene expression. Excess paraffin was trimmed and, then, total RNA was extracted from sectioned tissue blocks, using the absolutely RNeasy FFPE kit (QIAGEN, Germany). The integrity and size distribution of total RNAs were checked by $2 \%$ agarose gel electrophoresis with SYBR safe ${ }^{\mathrm{TM}}$, and two distinct ribosomal RNA bands were observed in each sample. The purity of RNAs was estimated by UV-spectrophotometry (Thermo Scientific, USA) in 10 uMTris-Cl buffer $(\mathrm{pH}=7.5)$. TheA260/280 ratio was 1.9 to 2.1 . 


\section{Reverse transcription}

Total RNAs extracted from FFPE tissue sections were reverse- transcripted in a final volume of 20 uL by the first-strand cDNA synthesis kit(Thermo, USA). To detect and exclude any interference by residual DNA contamination, polymerase chain reaction (PCR0 was performed with the same total RNA samples without reverse transcriptase. Products were electrophoresedon 2\% agarose gel.

\section{Real - time quantitative reverse transcription PCR}

Real-time quantitative RT-PCR amplification for SPHK1 and $\beta$-actin mRANs were performed using the 7500 real time PCR system (Applied Biosystem- USA),power SYBR Green PCR master mixes (Real $\mathrm{Q}$ plus 2x master Mix Green, AMPLIQON, Denmark), and they were run in duplicate $20 \mathrm{uL}$ reactions using $50 \mathrm{ng}$ of synthesized cDNA per reaction well, final concentration of primers was $5 \mathrm{pM}$. PCR cycling program was as follows: $95^{\circ} \mathrm{C}$ for 15 minutes for one cycle, $95^{\circ} \mathrm{C}$ for 20 seconds, $62.4^{\circ} \mathrm{C}$ for 30 seconds, and $72^{\circ} \mathrm{C}$ for 30 seconds for 45 cycles. To ensure that the resulting signals were derived from extracted RNAs, PCR also was performed fora similar control, but without the RT reaction (Figure $1)$.

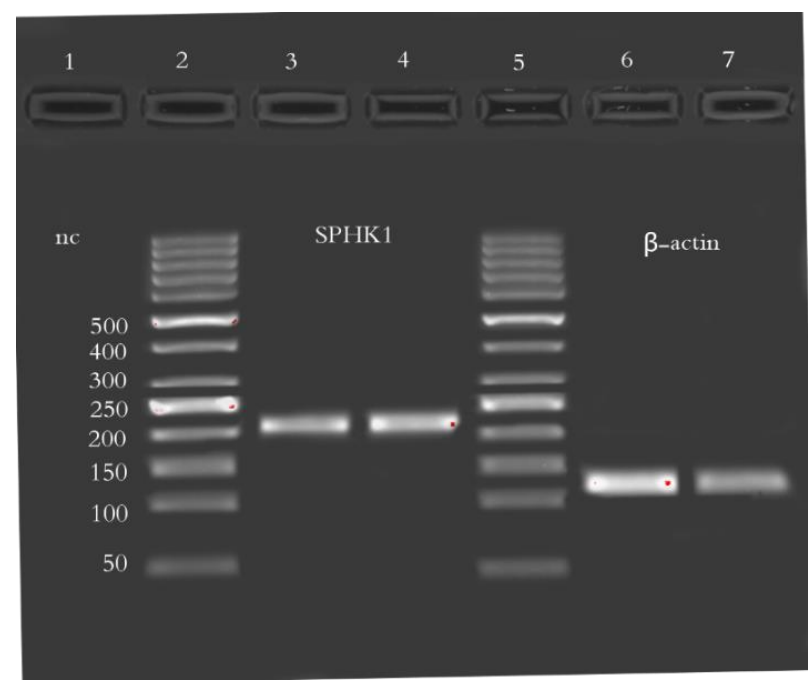

Figure 1.Real-time PCR products of SPHK1 and $\beta$-actin electrophoresedon $2 \%$ agarose gel in 80 constant voltage; from left to right 1: negative control;2, $5: 50 \mathrm{bp}$ ladder;3, 4: SPHK1 band(204 bp);6,7: $\beta$-actin band (120 bp)

REST384 version 2 (2006) software was used to obtain data and compare mean values among the groups.

\section{Primer Design}

According to Intron-spanning of SPHK1 gene, a pair of primers was designed by Perl-primer (v.1.1.19) software. To determine the quality of synthesized cDNA and normalization of PCR reaction; $\beta$-actin, as a house keeping gene, was used and previously designed (NCBI reference sequence NM 001101.3) (Table 1).

Table I. Information of Designed Primers

\begin{tabular}{clll}
\hline Gene & Primer Sequence $\left(5^{\prime}-3^{\prime}\right)$ & Tm $\left({ }^{\circ} \mathbf{C}\right)$ & Amplicon Size (bp) \\
\hline \multirow{2}{*}{$S P H K 1$} & F: CTTCCTTGAACCATTATGCTG & 62.4 & 204 \\
\multirow{2}{*}{$\begin{array}{l}\text { R:GCCGATACTTCTCACTCTC } \\
\text {-actin }\end{array}$} & $\begin{array}{l}\text { F: ACCACCTTCAACTCCATCATG } \\
\text { R: CTCCTTCTGCATCCTGTCG }\end{array}$ & 62.4 & 120 \\
\hline
\end{tabular}

Abbreviations: $\mathrm{F}$, forward primer, $\mathrm{R}$, reverse primer, Tm, annealing temperature; and bp, base pair

\section{Results}

To compare the expression level of SPHK1 in ER and $P R$ positive and negative Iranian breast cancer, and with their normal breast tissue, quantitative RT - PCR was performed. All RT-PCR reactions were repeated twice to minimize the experimental error. REST384- version 2 (2006) software was employed for data analysis. The current study data suggested a significant difference between SPHK1 expression level in negative receptor of estrogen and progesterone, compared with the positive receptors of breast cancer $(\mathrm{P}$-value $=0.0177)($ Figure 2$)$.
Comparison of BMI between the two tumor groups demonstrated that patients with negative receptors had higher BMI (> 25) than the patients with positive receptors (Figure 3).

The current study confirmed no statistically significant relationship between the expression level of SPHK1 in negative and positive normal breast tissue, compared to their tumor samples ( $\mathrm{P}$ values $=0.641$ and 0.48 ). 


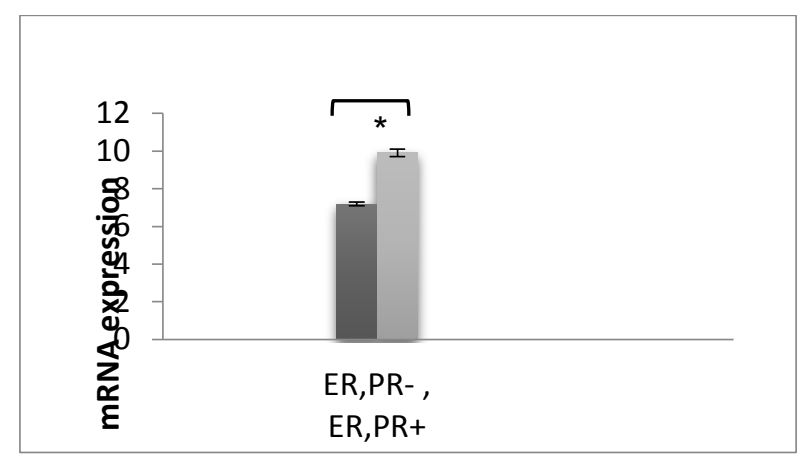

Figure 2.SPHKland $\beta$-actin expression levels in the studied groups; $P$-value $<0.05$

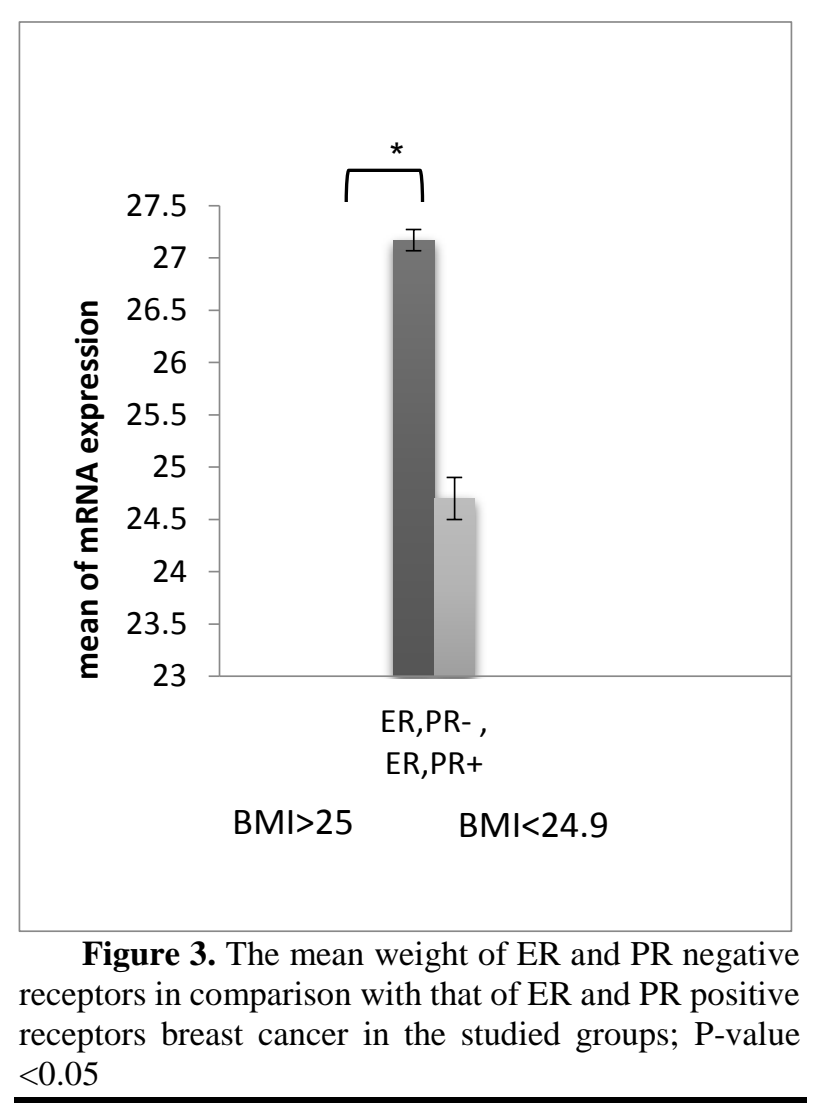

\section{Discussion}

Normal breast stroma tissue is changed into active stroma, during tumorigenesis. Stromal microenvironment of tumor cells is reported as a new factor in cancer progression. The metastatic rate of multiple cancers, including breast cancer, depends on the bilateral communication between tumor cells and their surrounding microenvironment $(22,23,24)$. SPHK1 and its product (S1P), known as biolipid microenvironment, have critical roles in regulation of cell proliferation, differentiation, and angiogenesis $(10,25)$. The level of SPHK1 and its product S1P is elevated in multiple cancers, including breast cancer, especially in ER and PR negative tumors (26). In this regard, a study in 2000 observed that SPHK1 displayed higher expression among ER negative breast cancer (27). In agreement with that, Longs et al. and Pyne et al. reported that expression of SPHKlhadno effective impact, and its inhibition might be of little use in ER- positive breast cancer treatment $(28,29)$, while high expression of $S P H K I$ is associated with shorter disease-specific survival in ER negative tumors (30). Also, further confirming results showed that SPHK1 expression increased up to four-fold in breast cancer, compared with the control groups (31).The current study confirmed the previous studies and showed a strong positive correlation between SPHK1 expression in ER and PR negative tumors in comparison with ER and PR positive breast cancer. Although the mechanisms of more highly SPHKl expression in negative receptors of breast tumor is unclear, it can be due to its higher proliferation activity (27), which is consistent with the anti-apoptotic effect of S1P (10). Similarity of the oncogenic lipid kinase (SPHK1) to leptin has led many studies done on the relationship between this gene, obesity, and breast cancer; although these mechanisms and pathways were linked with poor prognosis (32). Blachino et al., showed that fat tissue of obese females has high content of SPHK1, which induces proliferative response, offering that obesity maybe a factor for SPHK1 levels (33). More studies suggested that expression of SPHKland leptin receptor (LEPR) elevated in ER negative breast tumor with higher BMI $(34,35)$. Further investigations showed that leptin, as a prominent adipokine, has an essential role in breast tumor prognosis, advanced stage, and metastasis $(36,37,38)$. A new pathway suggested that leptin induces phosphorylation of STAT3 and SFK, and finally increasesSPHK1 mRNA and metastasis in ER negative breast cancer (32). Similarly, the current study results indicated that in ER negative breast cancer, SPHK1 is expressed more highly associated with the elevation of BMI. IHC assay of breast cancer showed that the major source of SPHK1 expression is carcinoma cells (10) that finally lead to produce S1P, which is the cause of angiogenesis (39), lymphangiogenesis (40), cell survival, and migration (41). As the expression of 
SPHK1 is associated with resistance to radiation and chemotherapies $(42,43)$, using SPHKI inhibitors and its specific analogs can be effective in the treatment of ER negative breast tumors. In this regard several studies in cancer cell lines and animal models were conducted $(21,44)$.

\section{Conclusion}

Overall, the results of the current study showed that $S P H K 1$ was expressed more highly in breast tumors, especially in negative receptors, and its expression can be associated with higher BMI in the patients; although further investigations are needed to find out its mechanisms as well as its subsequent influence on breast tumor.

\section{Acknowledgments}

Authors would like to thank the personnel and management of Afzalipour and Bahonar hospitals and Danesh Laboratory for their kind supports.

\section{References}

1. World Cancer Report. International Agency for research on Cancer. 2008Retrieved 2011-02-26.

2. Beckmann MW, Bani MR, Fasching PA, Strick $\mathrm{R}$ and Lux MP. Risk and risk assessment for breast cancer: molecular and clinical aspects. Maturitas 2007.20; 57(1): 56-60.

3. Mousavi SM, Montazeri A, Mohagheghi MA, Jarrahi AM, Harirchi I, Najafi M, Ebrahimi M. Breast cancer in Iran: an epidemiological review. Breast J 2007; 13(4):383-91.

4. Lal P, Tan LK, Chen B. Correlation of HER-2 status with estrogen and progesterone receptors and histologic features in 3,655 invasive breast carcinomas. Am J Clin Pathol 2005; 123(4): 541-6.

5. Blanco G, Holli K, Heikkinen M, Kallioniemi OP, Taskinen P. Prognostic factors in recurrent breast cancer: relationship to site of recurrence, disease- free interval, female sex steroid receptors, ploidy and histological malignancy grading. Br J Cancer 1990; 62(1): 142-6.

6. Marzec KA, Baxter R C, and. Martin JL. Targeting Insulin-Like Growth Factor Binding Protein-3 Signaling in Triple-Negative Breast Cancer. BoiMed Res Int 2015; 2015:638526.
7. Cuvillier O, Nava VE, Murthy SK, Edsall LC, Levade T, Milstien S, Spiegel S. Sphingosine generation, cytochrome c release, and activation of caspase-7 in doxorubicin-induced apoptosis of MCF7 breast adenocarcinoma cells. Cell Death Differ2001; 8(2):16271.

8. Herr DR, Chun J. Effects of LPA and S1P on the nervous system and implications for their involvement in disease. Curr Drug Targets2007;8 (1):155-67.

9. Spiegel S, Milstien S. Sphingosine-1-phosphate: an enigmatic signaling lipid. Nat Rev Mol Cell Biol2003; 4(5):397-407.

10. Shida D, Takabe K, Kapitonov D, Milstien S, Spiegel S. Targeting SphK1 as a new strategy against cancer. Curr Drug Targets 2008 Aug; 9(8):662-73.

11. Maceyka M, Spiegel S.Sphingolipid metabolites in inflammatory disease.Nature2014; 5;510(7503):58-67.

12. Pyne NJ, PyneS.Sphingosine 1-phosphate and cancer.Nat Rev Cancer 2010; 10(7):489-503.

13. Alshaker H, Sauer L, Monteil D, Ottavini S, Strivats S, Bohler T, Pchejetshki D. Therapeutic potential of targeting SK1 in human cancers.Adv Cancer Res 2013; 117:143-200.

14. Zheng XD, Zhang Y, Qi XW, Wang MH, Sun $\mathrm{P}$, Zhang $\mathrm{Y}$, et al. Role of Sphk1 in the malignant transformation of breast epithelial cells and breast cancer progression. Indian J Cancer 2014 Oct-Dec; 51(4):5249.

15. XiaP,GambleJR,WangL,PitsonSM,MorettiPA, WattenbergBW,D'AndreaRJ,Vadas MA et al. An oncogenic role of sphingosine kinase.CurrBiol2000; 10(23):1527-30

16. Ko P, Kim D, You E, Jung J, Oh S, Kim J et al. Extracellular Matrix Rigidity-dependent Sphingosine-1phosphate Secretion Regulates Metastatic Cancer Cell Invasion and Adhesion. Sci Rep 2016 Feb 15; 6:21564.

17. Tian H, Yu Z. Resveratrol induces apoptosis of leukemia cell line K562 by modulation of sphingosine kinase-1 pathway. Int J Clin Exp Phathol 2015 Mar 1; $8(3): 2755-62$.

18. Salama MF, Carroll B, Adada M, PuLkoskiGross M, Hannun YA, Obeid LM. A novel role of sphingosine kinase-1 in the invasion and angiogenesis of VHL mutant clear cell renal cell carcinoma. FASEB J $2015 \mathrm{Jul} ; 29(7): 2803-13$. 
19. Beach JA, Aspuria PJ, Cheon DJ, Lawenson K, Agadjanian H, Walsh CS. Sphingosine kinase 1 is required for TGF- $\beta$ mediated fibroblastto- myofibroblast differentiation in ovarian cancer. Oncotarget 2016 Jan 26; 7(4):4167-82.

20. Le ScolanE ,Pchejetski D,BannoY, DenisN, MayeuxP, Vainchenker $\mathrm{W}$ et al. Overexpression of sphingosine kinase 1 is an oncogenic event in erythroleukemic progression. Blood 2005;106(5):180816.

21. Zhao R, Milstien S, Zhou H, Spiegel S, Takabe $\mathrm{K}$ : Sphingosine-1-phosphateproduced by sphingosine kinase 1 promotes breast cancer progressionby stimulating angiogenesis and lymphangiogenesis. Cancer Res 2012 Feb 1; 72(3):726-735.

22. Wang E, NgalameY Panelli MC, NguyenJackson H, Deavers M, Mueller P, HuW, Savary CA et al. Peritoneal and subperitoneal stroma may facilitate regional spread of ovarian cancer. Clin Cancer Res 2005; 11(1):113-22.

23. Butcher DT, Alliston T, Weaver VM. A tense situation: forcing tumour progression.Net Rev Cancer 2009 Feb; 9(2):108-22.

24. Leventl KR, Yu H, Kass L, Lakins JN, Egeblad M, ErlerJT, Fong SF et al. Matrix crosslinking forces tumor progression by enhancing integrin signaling. cell 2009 Nov 25;139(5):891-906.

25. Shirai K, Kaneshiro T, Wada M, Furuya H, Bielawski J, Hannun YA, Obeid LM, et al. A role of sphingosine kinase 1 in head \& neck carcinogenesis. Cancer Prev Res (Phila). 2011 Mar; 4(3):454-62.

26. Tsuchida J, Nagahashi M, Nakajima M, Moro K, Tatsuda K, Ramanathan R Breast cancer sphingosine1-phosphate is associated with phospho-sphingosine kinase 1 and lymphatic metastasis.JSurgRes2016; 205(1):85-94.

27. Ruckhäberle E, Rody A, Engels K, Gaetje R, vonMinckwitz G, Schiffmann $S$ et al. Microarray analysis of altered sphingolipid metabolism reveals prognostic significance of sphingosine kinase 1 in breast cancer. Breast Cancer Res Treat 2008; 112(1):41-52.

28. Long JS, Edwards J, Watson C, Tovey S, Mair $\mathrm{KM}$, Schiff $\mathrm{R}$ et al. Sphingosine kinase 1 induces tolerance to human epidermal growth factor receptor 2 and prevents formation of a migratory phenotype in response to sphingosine 1-phosphate in estrogen receptor-positive breast cancer cells. Mol Cell Biol 2010 Aug; 30(15):3827-41.
29. Pyne NJ, Tonelli F, Lim KG, Long J, Edwards J, Pyne S: Targeting sphingosine kinase 1 in cancer. AdvBiolRegul 2012 Jan or:ץ, ;-38.

30. Ohotski J, Long JS, Orange C, Elsberger B, Mallon E, Doughty J.Expression of sphingosine 1phosphate receptor4 and sphingosine kinase 1 is associated with outcome in oestrogen receptor-negative breast cancer. Br J Cancer 2012; Apr 10; 106(8):1453-9.

31. French

KJ,SchrecengostRS,LeeBD,ZhuangY,SmithSN,EberlyJ L, YunJK,SmithCD.Discovery and evaluation of inhibitors of human sphingosine kinase.CancerRes2003; 63(18):5962-9.

32. Alshker H, Krell J, Frampton AE, Waxman J, BlyussO,Zaikin A. et al. Leptin induces upregulation of sphingosine kinase 1 in oestrogen receptor-negative breast cancer via Src family kinase-mediated, janus kinase 2-independent pathway. Breast Cancer Res 2014 Oct $25 ; 16(5): 426$.

33. Błachnio-

ZabielskaAU,PułkaM,BaranowskiM,NikołajukA,Zabiel skiP,Górska

M.et al.Ceramidemetabolismisaffectedbyobesityanddiabetesi nhumanadipose tissue.J Cell Physiol2012; 227(2):550-7.

34. Ruckhaberle E, Rody A, Engels K, Gaetje R, von Minckwitz G, Schiffmann S, Grosch S et al. Microarray analysis of altered sphingolipid metabolism reveals prognostic significance ofsphingosine kinase 1 in breast cancer. Breast Cancer Res Treat 2008 Nov; 112(1):41-52.

35. Blachnio-Zabielska AU, Pulka M, Baranowski M, Nikolajuk A, ZabielskiP,Gorska M. Ceramide metabolism is affected by obesity and diabetes in human adipose tissue. J Cell Physiol 2012 Feb; 227(2):550-7.

36. Miyoshi Y,FunahashiT, TanakaS, TaguchiT, TamakiY, Shimomura I et al High expression of leptin receptor mRNA in breast cancer tissue predicts poor prognosis for patients with high, but not low, serum leptin levels. Int J Cancer 2006; 118(6):1414-9.

37. MacciòA, MadedduC, GramignanoG, MulasC, FlorisC, Massa D et al. Correlation of body mass index and leptin with tumor size and stage of disease inhormone-dependent postmenopausal breast cancer preliminary results and the rapeuticimplications. JMol Med (Berl) 2010; 88(7):677-86.

38. Ishikawa M,Kitayama J, Nagawa H. Enhanced expression of leptin and leptin receptor (OB-R) in human breast cancer. Clin Cancer Res 2004; 10(13):4325-31. 
39. Visentin B, Vekich JA, Sibbald BJ, Cavalli AL, Moreno KM, Matteo RG et al. Validation of an antisphingosine-1-phosphate antibody as a potential therapeutic in reducing growth, invasion, and angiogenesis in multiple tumor lineages. Cancer Cell $2006 ; 9(3): 225-38$

40. Nagahashi M, Ramachandran S, Kim EY, Alle good JC, Rashid OM, Yamada A et al.Sphingosine-1phosphate produced by sphingosine kinase 1 promotes breast cancer progression by stimulating angiogenesis and lymphangiogenesis.CancerRes2012; 72(3):726-35.

41. Mukhopadhyay P, Ramanathan R, Takabe K.S1P promotes breast cancer progression by angiogenesis and lymphangiogenesis. Breast Cancer Manag2015; 4(5):241-244.
42. Sobue S, Lwasiki T, Suqisaki C, Nagatu K, Kikuchi R, Murakami M. QuantitativeRT-PCRanalysis of sphingolipid metabolic enzymes in acuteleukemia and myelodysplastic syndromes. Leukemia 2006 Nov; 20(11):2042-6.

43. Johnson KR, Johnson KY, Crellin HG, Ogretmen B, Boylan AM, Harley RA, et al. Immunohistochemical distribution of sphingosine kinase 1 in normal and tumor lung tissue. $\mathbf{J}$ Histochem Cytochem 2005 Sep; 53(9):1159-66.

44. TahaTA,KitataniK,El-Alwani M, Bielawski J, Hannun YA, Obeid LM. Loss of sphingosine kinase-1 activates the intrinsic pathway of programmed cell death: modulation of sphingolipid levels and the induction of apoptosis.FASEBJ2006; 20(3):482-4.

\section{How to Cite This Article:}

Nazouri AS, asadpour o, Dabiri S, Pourseyedi B, Lashkarizadeh Mr, Zeinalyneghad H. High Expression of Sphingosine Kinase 1 in Estrogen and Progesterone Receptors-Negative Breast Cancer. Iran J Pathol. 2017;12(3):218-224. 